\title{
Comparative study of spectroscopic properties of the low-lying electronic states of 2,4-pentadien-1-iminium cation and its $\mathrm{N}$-substituted analogues
}

\author{
ANJAN CHATTOPADHYAY \\ Department of Chemistry, Birla Institute of Technology and Science (BITS), Pilani -KK Birla Goa Campus, \\ Goa 403 726, India \\ e-mail: anjan@bits-goa.ac.in; anjan_chattopadhyay@yahoo.com
}

MS received 31 January 2012; revised 16 April 2012; accepted 22 May 2012

\begin{abstract}
Semiempirical and ab initio-based CI methods have been employed to study the low-lying electronic states of 2,4-pentadien-1-iminium cation and its $\mathrm{N}$-substituted analogues with electron-donating (methyl, isopropyl) and electron-withdrawing (fluoromethyl) groups on nitrogen. Variations of the dihedral angles $\left(\Gamma_{3}\right.$, $\left.\Gamma_{4}\right)$ of the ground state have given the global minima and global maxima at $\left(180^{\circ}, 180^{\circ}\right)$ and $\left(90^{\circ}, 0^{\circ}\right)$ conformations, respectively, with some exceptions in the case of fluoromethyl compound. Increase in the $+\mathrm{I}$ effect on nitrogen shifts the TICT conical intersection point away from the $90^{\circ}$ ( $\Gamma_{3}$ dihedral angle) value, when the $\Gamma_{4}$ value is kept fixed at $180^{\circ}$. Transition moment values of the allowed $\mathrm{S}_{0}\left(1 \mathrm{~A}_{\mathrm{g}}-\mathrm{like}\right) \rightarrow \mathrm{S}_{1}\left(2 \mathrm{~B}_{\mathrm{u}}-\right.$ like $)$ transitions are expectedly higher than the forbidden $\mathrm{S}_{0}\left(1 \mathrm{~A}_{\mathrm{g}}-\mathrm{like}\right) \rightarrow \mathrm{S}_{2}\left(2 \mathrm{~A}_{\mathrm{g}}-\right.$ like $)$ transitions by almost $5.6 \mathrm{D}$. Radiative lifetime values of the first excited states are calculated to be around 215 ps for all the four compounds. At $\left(180^{\circ}, 180^{\circ}\right)$ conformation the vertical excitation energy (VEE) between the $S_{0}$ and $S_{1}$ states of the 2,4-pentadieniminium cation is found to be $3.3 \mathrm{eV}$, which corresponds to the absorption wavelength value of roughly $375 \mathrm{~nm}$. The VEE value increases with substituents having $+\mathrm{I}$ effect on nitrogen, while for the fluoromethyl compound it is calculated to be around $2.85 \mathrm{eV}$. The energy gap between the first two excited singlet states is found to have the least value in the isopropyl-substituted compound, where the $\mathrm{S}_{2}$ state contains a huge contribution from the $\mathrm{HOMO}^{2} \rightarrow \mathrm{LUMO}^{2}$ configuration.
\end{abstract}

Keywords. Protonated Schiff base; configuration interaction; TICT conical intersection.

\section{Introduction}

Natural pigments like rhodopsin ${ }^{1}$ and bactereorhodopsin ${ }^{2}$ have driven lots of attention during the last few years. Their bio-optical activity is related to the ultrafast photoisomerization of the retinal Schiff base chromophores. Bactereorhodopsin consists of all-trans retinal connected to the lysine residue of a protein (consists of 248 amino acids) which forms an iminium ion or a protonated Schiff base (PSB). One of the two possible mechanisms of its photochemical processes proposed single all-trans to 13-cis isomerization, ${ }^{3}$ while the other one ${ }^{4-6}$ proposed all-trans to 13, 14-dicis double isomerizations. Several theoretical and experimental studies ${ }^{7-15}$ have been attempted on the configurations and spectroscopic properties of the Schiff base chromophores. Rotations about the two dihedral angles, $\Gamma_{13}$ and $\Gamma_{14}$ are the key factors behind the photochemical processes. The protonated Schiff base, 2, 4-pentadien-1-iminium cation, is usually chosen as the model compound for the retinal Schiff base. In this model compound, $\Gamma_{3}$ and $\Gamma_{4}$ are two dihedral angles (figure 1a), analogous to the earlier mentioned dihedral angles $\left(\Gamma_{13}\right.$ and $\left.\Gamma_{14}\right)$ in the retinal Schiff base, and the whole photochemical isomerization process of this species is controlled by them.

Major differences are noticed between the excited electronic states of the linear non-polar conjugated olefins and their isoelectronic polar protonated Schiff bases (PSB) of the corresponding polyenal. As an example, we can take the all-trans hexatriene (scheme 1) and pentadieniminium cation (scheme 2). In conjugated hexatriene the ground state $\left(1 \mathrm{~A}_{\mathrm{g}}\right)$ is found to be covalent. The lowest energy singlet excited state $\left(2 \mathrm{~A}_{\mathrm{g}}\right)$ is actually a two-electron excited $\left(\pi^{* 2}\right)$ covalent (diradical) state which originates due to the symmetry forbidden $\mathrm{S}_{0} \rightarrow \mathrm{S}_{1}$ transition and is consequently dark. The bright, ionic second excited singlet state $\left(1 \mathrm{~B}_{\mathrm{u}}\right)$ is the result of an allowed $\pi^{2} \rightarrow \pi \pi^{*}$ transition between $S_{0}$ and $S_{2}$ states (scheme 1). Initial excitation takes place from the $S_{0}$ state to the $S_{2}$ state, followed by an ultrafast (50-200 fs) ${ }^{16-20}$ relaxation to the $S_{1}$ state before again reaching to the ground state. So the first excited state $\left(S_{1}\right)$ collects some population from the $S_{2}$ state and controls the photochemical fate of the system. $S_{0}$ and $S_{1}$ in neutral polyenes are both covalent 
(a)

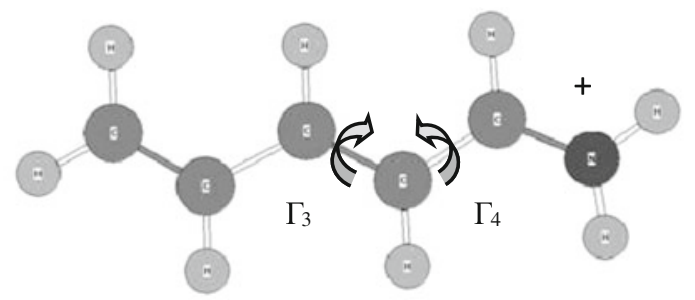

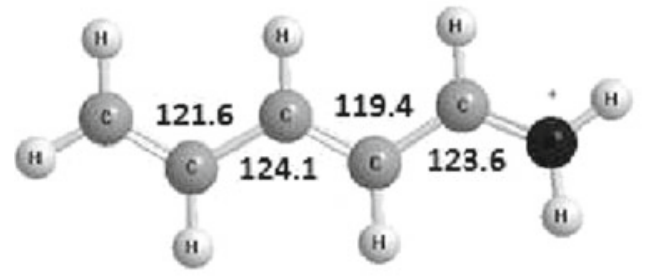

I

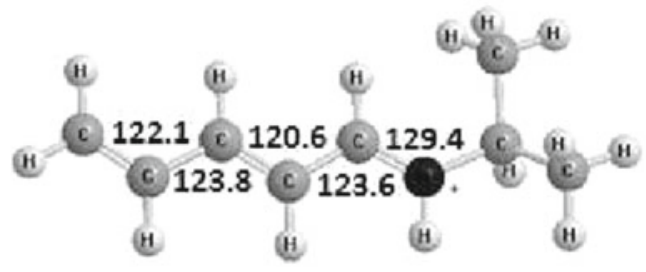

(b)

\section{III}

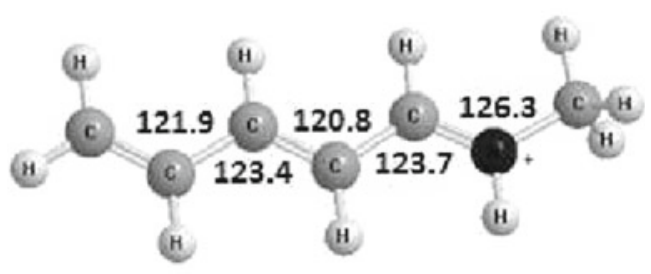

II

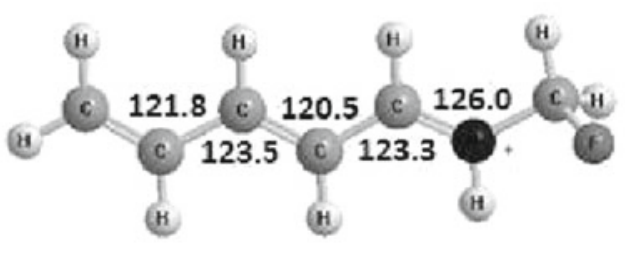

IV

Figure 1. (a) Dihedral angles are shown for the protonated Schiff base (I). (b) Ground state geometries of the four compounds at RHF level of calculation (other geometrical parameters are given in table 1).

states that belong to the same symmetry $\left(\mathrm{A}_{\mathrm{g}}\right)$; mixing and interaction between these two states are allowed. Consequently, all bonds have a $\pi$-bonding character in the ground state and the first excited singlet state and as a result, a well-defined planar minimum is there in the $\mathrm{S}_{1}$ state. These two covalent states can intersect, which

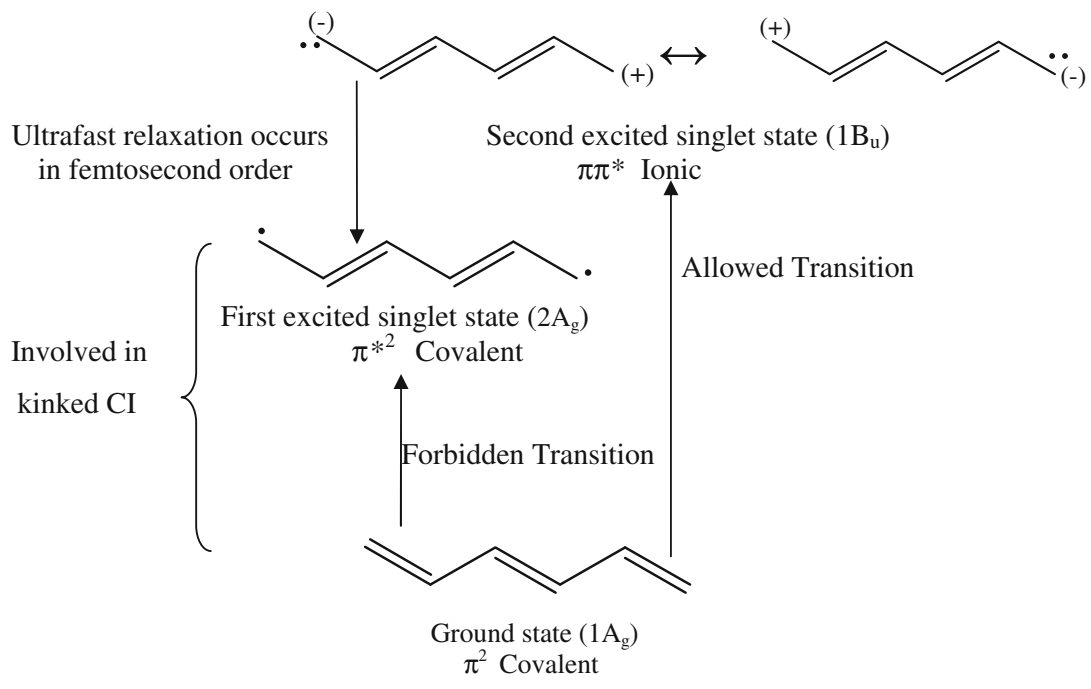

Neutral hexatriene system

Scheme 1. Photoexcitation of the ground state and its possible consequences in neutral hexatriene system. 


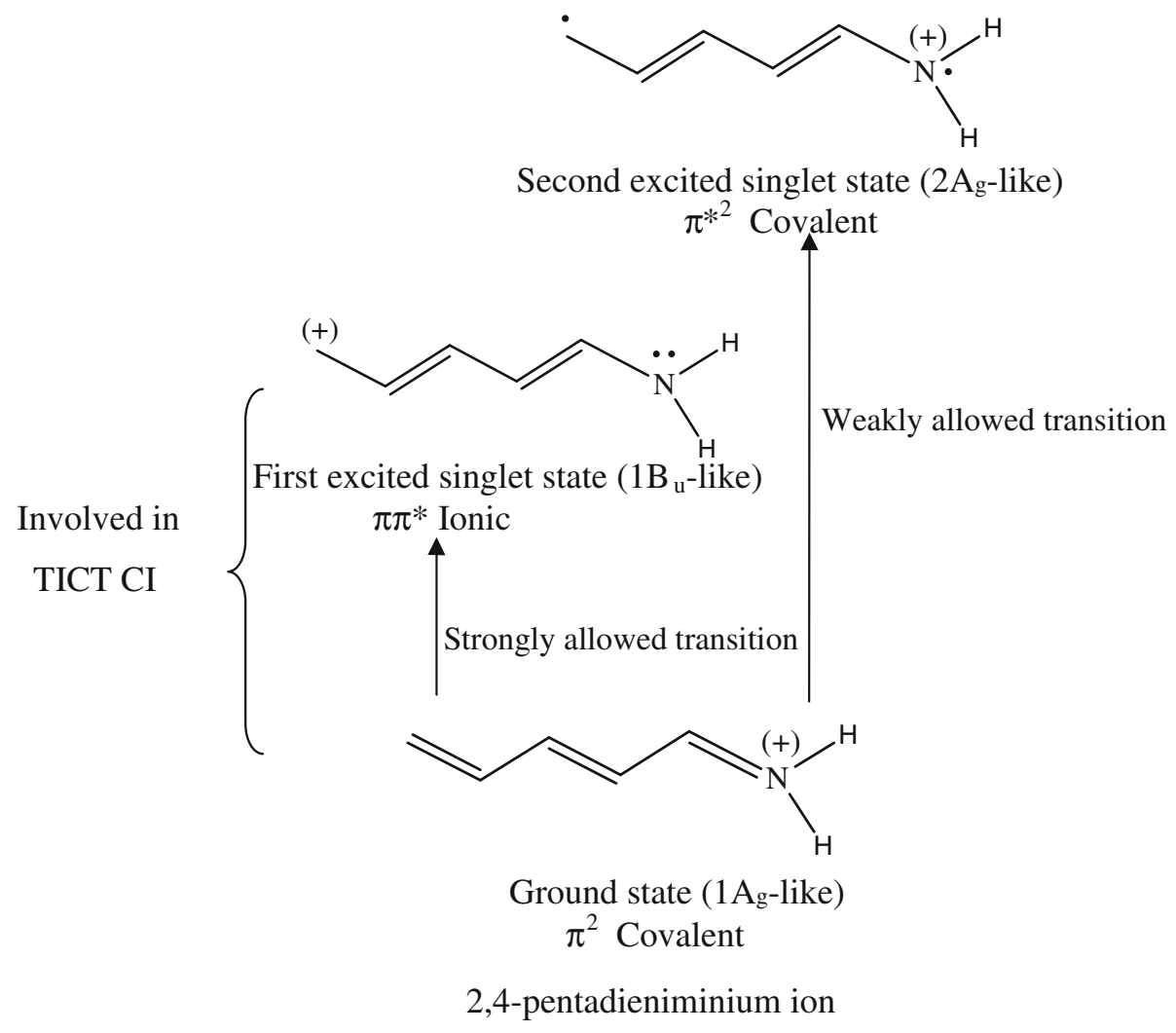

Scheme 2. Photoexcitation of the ground state and its possible consequences in polar 2,4-pentadieniminium ion.

is due to the degeneracy of these two electronic states arising out from a three-centre three electron bond in an out-of-plane triangular structure, commonly known as kinked CI. ${ }^{21}$

On the contrary, in pentadieniminium ion, the charge transfer $\pi \pi^{*}\left(1 \mathrm{~B}_{\mathrm{u}}\right.$-like $)$ state will be more stabilized in comparison to the $\pi^{* 2}\left(2 \mathrm{~A}_{\mathrm{g}}\right.$-like) state (scheme 2$)$. This is due to the stronger electron-withdrawing effect of $\mathrm{NH}_{2}^{+}$group than $\mathrm{CH}_{2}$. This will swap the order of the two low-lying excited singlet states. The first excited state will not interact with the ground $\left(1 \mathrm{~A}_{\mathrm{g}}\right.$-like) state due to the symmetry factor. Now the planar structure of this state is not necessarily a minimum as all bonds are not having $\pi$-bonding character anymore. This results in a barrierless process of one-bond flip (OBF) ${ }^{22-25}$ around a single bond $(\mathrm{C}-\mathrm{C})$ in the $\mathrm{S}_{1}$ state which was originally a double bond $(\mathrm{C}=\mathrm{C})$ in the ground state. Neutral polyenes are unlikely to do so as all bonds have a $\pi$-bonding character in the first excited singlet state and will involve huge barriers. During the rotation process, the excited state $\left(\mathrm{S}_{1}\right)$ becomes stabilized, whereas the ground state energy increases due to the rotation about a double bond. At $90^{\circ}$ torsion angle their energies will match and a conical intersection of these two surfaces will take place. Consequently, a radiationless transition occurs to the ground state. This intersection is commonly known as the twisted intramolecular charge transfer conical intersection (TICT CI). The molecule now comes to a local maximum at the ground state and then ultimately it comes to one of the stable ground state minima. As soon as the photon is absorbed, this ultrafast process happens spontaneously almost giving no chance for other competing processes, such as, fluorescence $\left(10^{-8} \mathrm{~s}\right)$, to take place. A high quantum yield $(65 \%)$ is obtained in case of retinal in rhodopsin.

In this present work, we have studied the ground state and the excited states of 2,4-pentadien-1-iminium ion and its $\mathrm{N}$-substituted analogues through introduction of some electron-donating and electron-withdrawing groups on the iminium nitrogen by replacing one hydrogen. Interesting results are expected to be observed due to the change of the position of the TICT conical interaction, and this is certainly going to affect the ultrafast dynamics of their barrierless rotations. Placing a negative counterion close to the positive chromophore ion and substitution of hydrogens by different groups on the long-chain carbon atoms have already been found ${ }^{22,23,26,27}$ to change the stability of the singlet (S0, $\mathrm{S} 1, \mathrm{~S} 2)$ states and subsequently the conical intersection position. Four compounds (figure 1b) are chosen 
for this present investigation, which includes the simple protonated Schiff base (I) and its analogues resulting due to the substitutions by methyl (II), isopropyl (III) and fluoromethyl (IV) groups on nitrogen. Some theoretical studies on PSB (I) have been earlier reported at different level of calculations. AM1/CISD, MP4, MRCI calculations have been carried out by Dobado and Nonella, ${ }^{25}$ while MNDO-CI method has been applied by two different groups. ${ }^{28,29}$ Few years back, Tavernelli et al. ${ }^{30}$ has employed the TDDFT technique to study the geometry and excitation energy values of this (I) species.

Inclusion of methyl and isopropyl groups are supposed to neutralize the positive charge on nitrogen, while fluoromethyl group is likely to increase the same through its -I effect. Position of the positive charge on the above mentioned systems depend on the nature of the electronic state; it is closer to the carbon-tail for the charge-transfer state, while for the covalent states, it is located on the nitrogen-head (N-head). The ground state stability will be highly influenced due to the $+\mathrm{I}$ and $-\mathrm{I}$ effects as the covalent ground state carries a positive charge on the nitrogen. Transition moment calculations of the first two low-lying transitions of these systems are reported in this present work; this includes the values of some important spectroscopic parameters, such as Einstein's coefficient (A), oscillator strengths (length form and velocity form) and vertical excitation energies. Radiative lifetime values are also calculated for the excited singlet states.

\section{Computational details}

In this present work, semiempirical as well as $a b$ initio-based quantum mechanical studies are attempted on the low-lying electronic states of the above mentioned molecules. Configuration interaction technique with semiempirical-based Hamiltonian (PM3) has been employed in MOPAC for optimization of geometries and studies of the points on the potential energy surfaces. Stewart's Parametric Method Number 3(PM3) is based ${ }^{31,32}$ on the neglect of Differential Diatomic Overlap (NDDO) integral approximation. This PM3 Hamiltonian has been used with multielectron configuration interaction technique (MECI) in this present study. Restricted-Hartree-Fock method (RHF) and CISD technique have also been used for more accurate geometry optimizations through the GAMESS ${ }^{33}$ suite of programs. The SCF-MOs generated by the RHF method are used for the configuration interaction calculations. For CI studies, Graphical Unitary Group Approach (GUGA) $\mathrm{CI}^{34-37}$ has been employed.
This method is based on Brooks and Schaefer's unitary group program, which was modified to run within GAMESS using Davidson's eigenvector method, ${ }^{38}$ written by Steve Elbert. ${ }^{39}$ Graphical Unitary Group Approach (GUGA) specifies configurations using the Distinct Row Table (DRT) by \$CIDRT group in GAMESS. This DRT defines the Hamiltonian matrix by means of loop-driven algorithm. For example, in system I, inner 12 electrons are taken into frozen core (not correlated), while 32 electrons are kept doubly occupied in 16 orbitals $(\mathrm{NDOC}=16)$. These electrons are excited to the virtual orbitals and the excitation level is selected to be 2 (EXCIT $=2$ ), which gives rise to singles and doubles CI. Transition moment and oscillator strength ${ }^{40-42}$ calculations are based on this GUGA CI code. Radiative lifetime of the first excited state has been determined from the Einstein's coefficient, $A_{21}$. It is well-known that Einstein's coefficient $\left(A_{21}\right)$ is defined in terms of total rate of spontaneous emission $\left(\mathrm{W}_{21}\right)$ from an upper level (2) to a lower level (1) and number of atoms $\left(\mathrm{n}_{2}\right)$ in the upper level,

$$
\mathrm{W}_{21}=\mathrm{A}_{21} \mathrm{n}_{2}
$$

and it's value is reciprocal of the spontaneous radiative lifetime ( $\mathrm{t}$ ) of level 2, if level 2 decays through radiative emission to level 1.

$$
\mathrm{A}_{21}=1 / \mathrm{t} \text {. }
$$

Einstein's coefficient (A) and oscillator strength values are obtained from the output of the transition moment calculations.

\section{Results and discussions}

Optimization results of the ground state geometries at the RHF and CISD level using 6-311G (d,p) and 6$31 \mathrm{G}$ basis sets, respectively, are shown in table 1 . It can be noticed that the bond distances of the double bonds $(\mathrm{C} 3-\mathrm{C} 4, \mathrm{C} 5-\mathrm{N})$ are further reduced from the values of the protonated Schiff base (I), when more efficient electron donating groups (II and III) are introduced on nitrogen. On the other hand, increase in the single bond lengths (C2-C3 and C4-C5) can be noticed. Effect of these $+\mathbf{I}$ groups decreases as we go further away from the positive charge. Electron-withdrawing group (IV) increases positive charge on nitrogen; thus destabilizes the system and slight deviation from the planar geometry is observed. As mentioned earlier, equilibrium geometries of the excited states are not planar as there will be no mixing of the ground state and the first excited state due to the symmetry factor, and consequently all bonds are not having $\pi$-bonding 
Table 1. Geometric parameters (dihedral angles, bond lengths) of the ground states of the four compounds.

\begin{tabular}{lcccccccc}
\hline Compounds & Method & $\Gamma_{3}$ & $\Gamma_{4}$ & $\mathrm{C} 1-\mathrm{C} 2$ & $\mathrm{C} 2-\mathrm{C} 3$ & $\mathrm{C} 3-\mathrm{C} 4$ & $\mathrm{C} 4-\mathrm{C} 5$ & $\mathrm{C} 5-\mathrm{N}$ \\
\hline $\mathbf{I}$ & RHF/6-311G** & 180 & 180 & 1.327 & 1.440 & 1.353 & 1.409 & 1.292 \\
& CISD/6-31G & 180 & 180 & 1.349 & 1.448 & 1.368 & 1.421 & 1.312 \\
& RHF/6-31G*a & 180 & 180 & 1.332 & 1.442 & 1.356 & 1.410 & 1.295 \\
& TDDFT $^{\mathrm{b}}$ & 180 & 180 & 1.391 & 1.520 & 1.357 & 1.476 & 1.353 \\
II & RHF/6-311G** $^{*}$ & 180 & 180 & 1.326 & 1.445 & 1.347 & 1.418 & 1.284 \\
III & RHF/6-311G** & 180 & 180 & 1.325 & 1.447 & 1.344 & 1.424 & 1.281 \\
IV & RHF/6-311G** & 180 & 177 & 1.328 & 1.439 & 1.350 & 1.410 & 1.292 \\
\hline${ }^{\mathrm{a}}[25],{ }^{\mathrm{b}}[30]$ & & & & & & & &
\end{tabular}

nature in the $S_{1}$ state. For example, optimization of the first excited state of compound III at the PM3/CI level leads to a minimum energy geometry with $\Gamma_{3}$ value close to $180^{\circ}$ and $\Gamma_{4}$ value of $100^{\circ}$. Single bond lengths are quite higher $(1.502 \AA)$ in comparison to the ground state, indicating poor delocalization of electronic charge. Similarly, for compound II and I, $\Gamma_{4}$ values are higher, $141^{\circ}$ and $156^{\circ}$, respectively, while for the fluoromethyl compound deviation from $180^{\circ}$ is very small for this dihedral angle. It has been found that in all

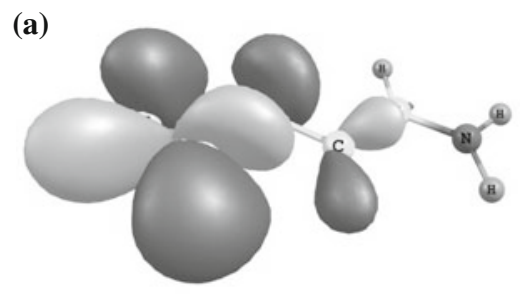

HOMO-2

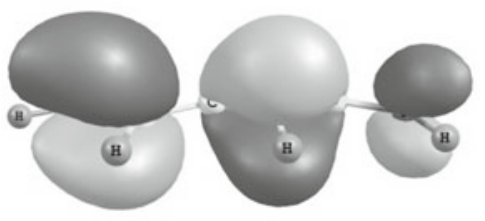

HOMO

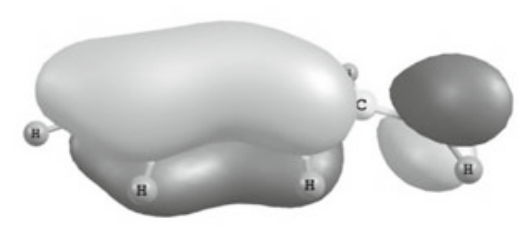

HOMO-1

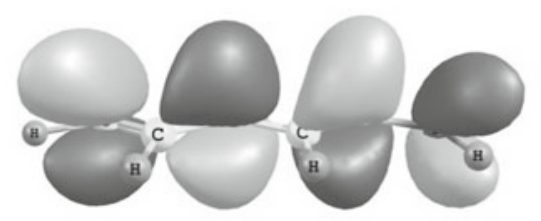

LUMO

(b)

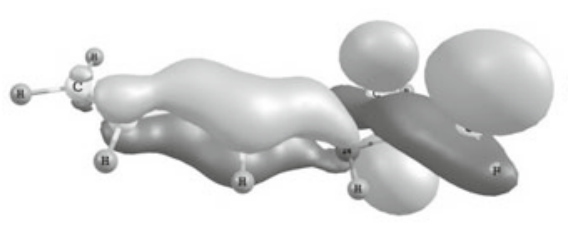

HOMO-2

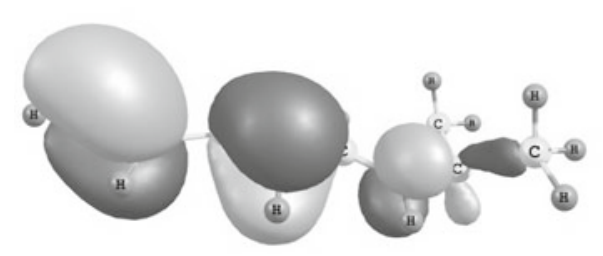

HOMO

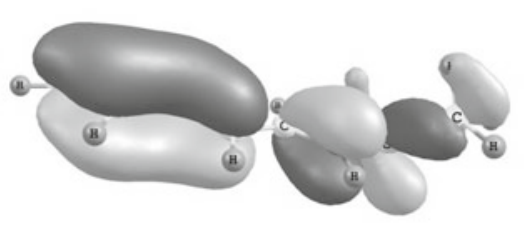

HOMO-1

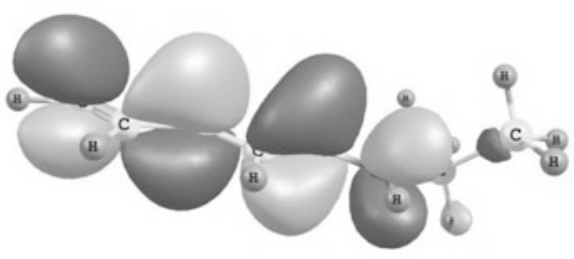

LUMO

Figure 2. Some important molecular orbitals of the (a) protonated Schiff base (I) and (b) its isopropyl-substituted derivative (III) at their respective ground state geometries. 
these cases the energy difference between the optimized ground and excited state geometry is almost $2 \mathrm{eV}$.

Some important molecular orbitals (HOMO, HOMO1, HOMO-2, LUMO) derived from the Hartree-Fock calculations at the ground state geometry of $\mathbf{I}$ and its isopropyl-substituted analogue (III) are shown in figure 2. These are the key MOs involved in the formation of the first three excited singlet states $\left(\mathrm{S}_{1}\right.$, $\mathrm{S}_{2}, \mathrm{~S}_{3}$ ). First four roots of the GUGA-CI calculation

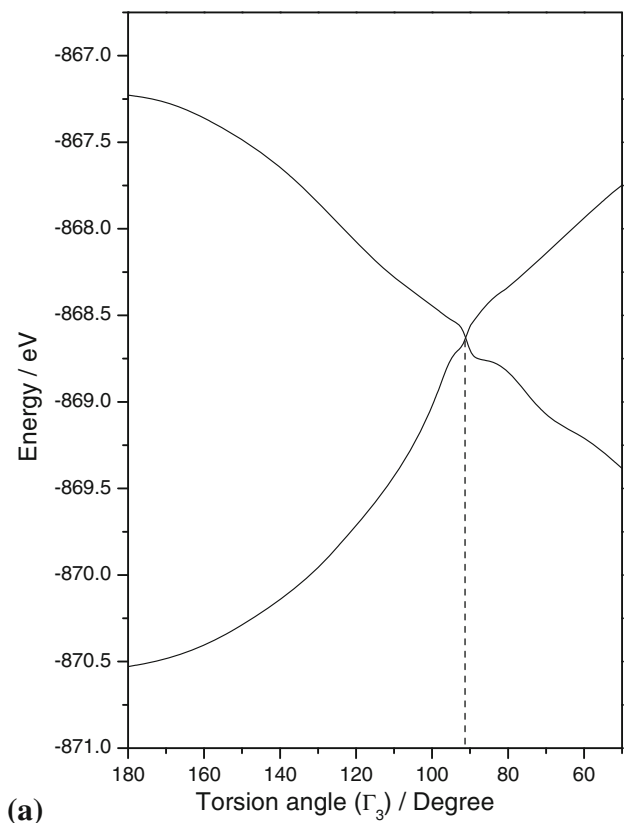

(a)

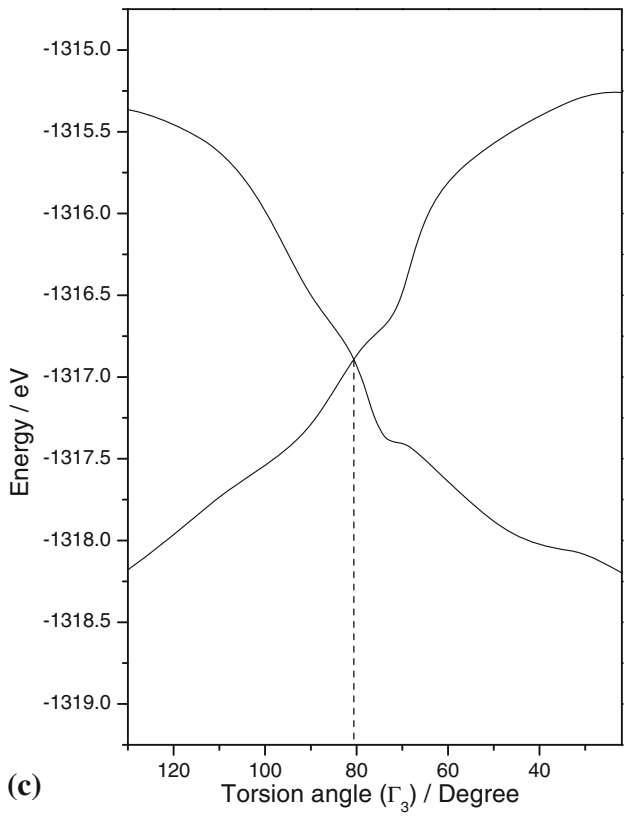

at the ground state geometry has been analysed for all the molecules. The first excited state $\left(S_{1}\right)$ is arising due to the HOMO $\rightarrow$ LUMO transition, while the $\mathrm{S}_{2}$ state which is actually a biradical species is having major contributions from HOMO- $\rightarrow$ LUMO and $\mathrm{HOMO}^{2} \rightarrow \mathrm{LUMO}^{2}$ configurations. The next excited singlet state has dominant contribution from HOMO$2 \rightarrow$ LUMO. Similar nature of the excited states has also been predicted by other level of studies ${ }^{25,28,30}$ on the

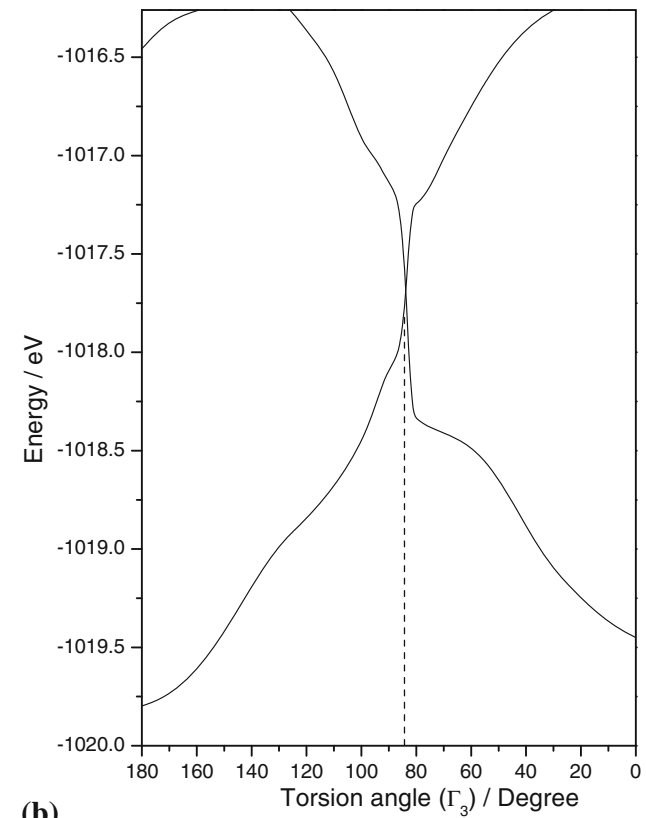

(b)

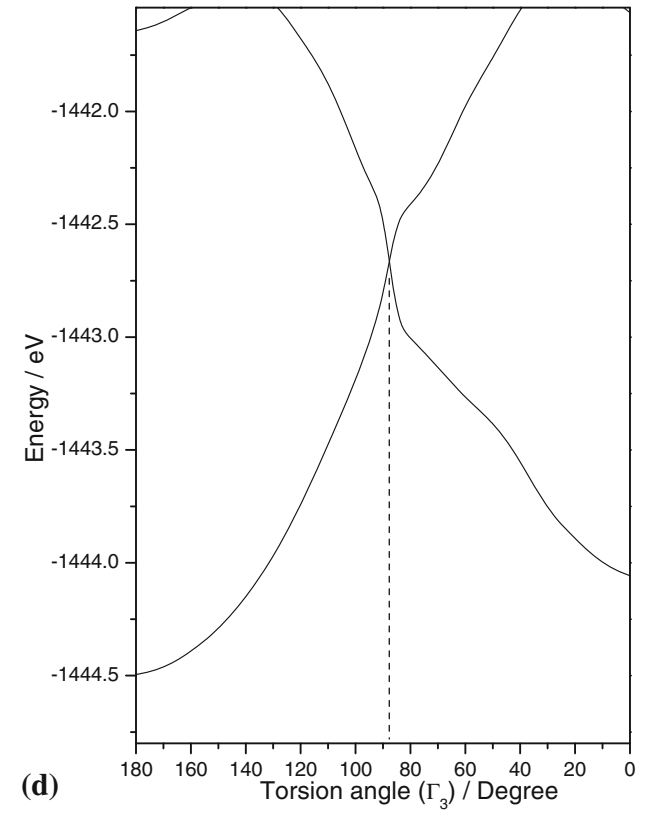

Figure 3. (a) Potential energy curves of the $S_{0}$ and $S_{1}$ states of compound $\mathbf{I}$ with variation of $\Gamma_{3}$ torsion angle, keeping $\Gamma_{4}$ fixed at $180^{\circ}$. (b) Potential energy curves of the $S_{0}$ and $S_{1}$ states of compound II with variation of $\Gamma_{3}$ torsion angle, keeping $\Gamma_{4}$ fixed at $180^{\circ}$. (c) Potential energy curves of the $S_{0}$ and $S_{1}$ states of compound III with variation of $\Gamma_{3}$ torsion angle, keeping $\Gamma_{4}$ fixed at $180^{\circ}$. (d) Potential energy curves of the $S_{0}$ and $S_{1}$ states of compound IV with variation of $\Gamma_{3}$ torsion angle, keeping $\Gamma_{4}$ fixed at $180^{\circ}$. 
protonated Schiff base (I). Couple of interesting points related to these higher-energy $\mathrm{CI}$ roots are required to be mentioned here; one is the amount of $S_{1}-S_{2}$ energy gap and the other is the contributions of HOMO$1 \rightarrow$ LUMO and $\mathrm{HOMO}^{2} \rightarrow \mathrm{LUMO}^{2}$ configurations in $\mathrm{S}_{2}$. The dominance of the latter configuration increases from I to III, while the above mentioned energy gap decreases in the same direction. This is expected, as more electron-donating groups will reduce the positive charge on nitrogen and to some extent can make it comparable to the neutral hexatriene system, where the order of the ionic $S_{1}$ state and covalent radicaltype $S_{2}$ state is reversed. So the $S_{2}$ energy level is very close to its immediate higher-energy singlet state in isopropyl-substituted compound (III) with an increased contribution from $\mathrm{HOMO}^{2} \rightarrow \mathrm{LUMO}^{2}$ configuration in it. However, it is quite obvious that accumulation of huge amount of electron cloud on the positively charged nitrogen can only swap the order of these two states and can give rise to situation similar to the neutral conjugated triene system.

Potential energy curves of the ground states and the first excited states have been plotted (figures $3 \mathrm{a}-\mathrm{d}$ ) for all the four compounds by varying the $\Gamma_{3}$ value $\left(0^{\circ}\right.$ to $\left.180^{\circ}\right)$ and keeping the $\Gamma_{4}$ value fixed at $180^{\circ}$. Energy values for all these systems have been reported at the PM3/CI level of studies. It has been found that the $\Gamma_{3}$ value corresponding to the conical intersection point has been shifted away from the $90^{\circ}$ value of 2,4-pentadien-1-iminium cation as electrondonating groups are substituted on nitrogen. This can be explained on the basis of the $+\mathrm{I}$ effect of methyl and isopropyl groups, which stabilizes the covalentground state, while the first excited state is destabilized. This certainly increases the $S_{0}-S_{1}$ energy gap and consequently the conical intersection point shifts towards the right-hand side in comparison to $\mathbf{I}$. The $\Gamma_{3}$ value corresponding to the TICT CI position of electron-withdrawing fluoro methyl substituted compound (IV) remains almost at the same position as compound $\mathbf{I}$. Effect of these different types of substituents on the nitrogen is quite comparable to the countercharge effect on iminium ions described by Garavelli. ${ }^{22}$ It has been reported that if the countercharge is placed at the central position, photoisomerization process is enhanced and ultrafast radiationless decay channels are favoured. On the contrary, placing the counter ion on $\mathrm{N}$-head or C-tail positions shifts the conical intersection point away from the $90^{\circ}$ mark and inhibits the ultrafast process. So it is quite obvious in our case that substitution of methyl or isopropyl groups will not favour the photoisomerization process. However, the effect of fluoromethyl group is not that significant; this is due to the weak -I effect of this group. May be a stronger electron-withdrawing group, such as trifluoromethyl, will have more pronounced effect on shifting the conical intersection point.

Some of the important points on the PES are studied through variations of the two dihedral angles, represented as $\left(\Gamma_{3}, \Gamma_{4}\right)$ throughout this work. In general, four distinct energy maxima are found on the PES of the ground state, and at least two energy minima have been obtained (table 2a) including the global energy minimum at all-trans $\left(180^{\circ}, 180^{\circ}\right)$ conformation. It can be noticed that the energy maxima at $\left(90^{\circ}, 90^{\circ}\right)$ corresponds to the global energy maxima for the first three compounds (I, II, III), while $\left(90^{\circ}, 0^{\circ}\right)$ conformation corresponds to the maximum energy of compound IV. First excited state energy values are also compared at the same level of theory. Comparison (table 2b) of the energy values of compound III and I is giving clear indication that excited state geometry is destabilized when strong electron-donating groups are present.

Transition moment calculations have given some interesting insights in the excited state properties of these four molecules. Using GUGA-CI code in

Table 2a. Comparison of the ground state energies (in kcal $/ \mathrm{mol}$ ) at different geometries $\left(\Gamma_{3}, \Gamma_{4}\right)$.

\begin{tabular}{|c|c|c|c|c|c|c|c|}
\hline \multirow[b]{2}{*}{$\left(\Gamma_{3}, \Gamma_{4}\right)$} & \multicolumn{4}{|c|}{ I } & \multirow{2}{*}{$\frac{\text { II }}{\mathrm{PM} 3 / \mathrm{CISD}}$} & \multirow{2}{*}{$\frac{\text { III }}{\mathrm{PM} 3 / \mathrm{CISD}}$} & \multirow{2}{*}{$\frac{\text { IV }}{\text { PM3/CISD }}$} \\
\hline & PM3/CISD & $\mathrm{AM} 1 / \mathrm{CISD}^{\mathrm{a}}$ & $\mathrm{MP}^{\mathrm{a}}$ & $\mathrm{MRCI}^{\mathrm{a}}$ & & & \\
\hline$\left(180^{\circ}, 180^{\circ}\right)$ & 0 & 0 & 0 & 0 & 0 & 0 & 0 \\
\hline$\left(90^{\circ}, 0^{\circ}\right)$ & 50.4 & 35.5 & 62 & 62.2 & 36.9 & 45.4 & 63.5 \\
\hline$\left(90^{\circ}, 180^{\circ}\right)$ & 40.1 & 33.5 & 59.4 & 59.6 & 40.2 & 43.6 & 38.5 \\
\hline$\left(180^{\circ}, 90^{\circ}\right)$ & 21.3 & 10.2 & 17.5 & 19.9 & 5.6 & 6.7 & 17.6 \\
\hline$\left(90^{\circ}, 90^{\circ}\right)$ & 58.5 & 48.3 & 108.9 & 96.7 & 46.1 & 63.4 & 47.9 \\
\hline$\left(0^{\circ}, 0^{\circ}\right)$ & 57.0 & 48.8 & 47 & 48.9 & 36.2 & 48.2 & 42.1 \\
\hline$\left(180^{\circ}, 0^{\circ}\right)$ & 8.0 & 2.8 & 6.5 & 6.6 & 2.5 & 8.0 & 18.8 \\
\hline$\left(0^{\circ}, 180^{\circ}\right)$ & 3.8 & 3.0 & 4.7 & 4.0 & 1.1 & 8.8 & 2.8 \\
\hline$\left(0^{\circ}, 90^{\circ}\right)$ & 20.2 & 10.1 & 18.8 & 21.4 & 10.7 & 21.7 & 8.4 \\
\hline
\end{tabular}


Table 2b. Comparison of excited state energies ( $\mathrm{kcal} / \mathrm{mol})$ of compounds I and III at different geometries $\left(\Gamma_{3}, \Gamma_{4}\right)$.

\begin{tabular}{|c|c|c|c|c|}
\hline \multirow[b]{2}{*}{$\left(\Gamma_{3}, \Gamma_{4}\right)$} & \multicolumn{3}{|c|}{ I } & \multirow{2}{*}{$\frac{\text { III }}{\text { PM3/CISD }}$} \\
\hline & PM3/CISD & $\mathrm{AM} 1 / \mathrm{CISD}^{\mathrm{a}}$ & $\mathrm{MRCI}^{\mathrm{a}}$ & \\
\hline $90^{\circ}, 0^{\circ}$ & -36.34 & -3.4 & -38.3 & -2.48 \\
\hline $180^{\circ}, 180^{\circ}$ & 0.00 & 0.0 & 0.0 & 0.0 \\
\hline $90^{\circ}, 180^{\circ}$ & -25.30 & -1.6 & -36.3 & -8.37 \\
\hline $180^{\circ}, 90^{\circ}$ & -1.25 & 18.0 & -2.3 & -17.5 \\
\hline $90^{\circ}, 90^{\circ}$ & -1.61 & 0.0 & 2.0 & 15.3 \\
\hline $0^{\circ}, 0^{\circ}$ & 53.74 & 50.1 & 45.9 & 65.9 \\
\hline $180^{\circ}, 0^{\circ}$ & -6.47 & 1.3 & -0.5 & -0.2 \\
\hline $0^{\circ}, 180^{\circ}$ & 3.46 & 3.2 & -1.4 & 13.5 \\
\hline $0^{\circ}, 90^{\circ}$ & -3.14 & 19.0 & -1.8 & 13.5 \\
\hline
\end{tabular}

${ }^{\mathrm{a}}[25]$

GAMESS, oscillator strength, transition moment and Einstein's coefficient values corresponding to the lowlying transitions $\left(\mathrm{S}_{0} \rightarrow \mathrm{S}_{1}, \mathrm{~S}_{0} \rightarrow \mathrm{S}_{2}\right)$ have been predicted (table 3). Radiative lifetime values of the first excited states $\left(S_{1}\right)$ for all the compounds calculated from the Einstein's coefficient (A) values are around 215 ps. These lifetime values are much higher than the observed femtosecond order dynamics of barrierless rotation of the excited state of retinal Schiff base which opens up the non-radiative decay channel through conical intersection. However, the competing radiative processes may have more importance in methyl and isopropyl-substituted compounds, as the quantum yield of the nonradiative process is expected to be less in these cases due to the slow rate of photoisomerization. As expected, the transition moment and oscillator strength values are comparatively lower for the $S_{0} \rightarrow S_{2}$ transition due to the forbidden nature of transition between the two states of same symmetry $\left(1 \mathrm{~A}_{\mathrm{g}}-\right.$ like $\rightarrow 2 \mathrm{~A}_{\mathrm{g}}-$ like $)$; consequently, the lifetime values of the $S_{2}$ states are comparatively higher. For the protonated Schiff base (I) and the fluoromethyl compound (IV), radiative lifetime values of their second excited singlet states are found to be $2.4 \mathrm{~ns}$, while the methyl (II) and the isopropyl (III) compounds are having slightly lower values $(\sim 2.2 \mathrm{~ns})$ for the same. It must be remembered that the lifetime of the $S_{2}$ state is very low for non-polar conjugated hexatriene system and it increases the population of the $S_{1}$ state rapidly through $\mathrm{S}_{2} \rightarrow \mathrm{S}_{1}$ transition. The $+\mathbf{I}$ effect of the methyl (II) and the isopropyl (III) groups reduces the positive charge on nitrogen; thus making it comparable to the neutral hexatriene system, to some extent; however, expectedly there is a huge difference in the order of the lifetime (ns) of the $\mathrm{S}_{2}$ states between these systems (II, III) and the non-polar conjugated triene system (fs).

Low-lying vertical excitation energies between $S_{0}$ and $S_{1}$ states are computed at two different geometries and compared with other reported values. At $\left(180^{\circ}, 180^{\circ}\right)$ conformation the absorption wavelength is

Table 3. Transition properties of some low-lying transitions.

\begin{tabular}{|c|c|c|c|c|c|c|c|c|c|}
\hline \multirow[b]{2}{*}{ Transition } & \multirow[b]{2}{*}{ Compound } & \multicolumn{4}{|c|}{ Transition moment (D) } & \multicolumn{2}{|c|}{$\begin{array}{l}\text { Oscillator } \\
\text { strength }\end{array}$} & \multirow{2}{*}{$\begin{array}{l}\text { Einstein's } \\
\text { coefficient }\end{array}$} & \multirow{2}{*}{$\begin{array}{l}\text { Radiative } \\
\text { lifetime ( } \mathrm{s}\end{array}$} \\
\hline & & $\mu$ & $\mu_{\mathrm{x}}$ & $\mu_{\mathrm{y}}$ & $\mu_{\mathrm{z}}$ & $f_{L}$ & $f_{V}$ & & \\
\hline \multirow{4}{*}{$\begin{array}{l}1 \mathrm{~A}_{\mathrm{g}} \text {-like } \rightarrow \\
1 \mathrm{~B}_{\mathrm{u}} \text {-like }\end{array}$} & I & 7.281 & -7.279 & -0.160 & -0.001 & 1.618 & 0.266 & $4.5540(+9)$ & $219(-12)$ \\
\hline & II & 7.543 & -7.523 & -0.556 & -0.004 & 1.695 & 0.448 & $4.5380(+9)$ & $220(-12)$ \\
\hline & III & 7.980 & -7.902 & -0.921 & 0.613 & 1.835 & 0.458 & $4.6046(+9)$ & $217(-12)$ \\
\hline & IV & 7.611 & -7.573 & -0.335 & 0.679 & 1.735 & 0.428 & $4.7017(+9)$ & $213(-12)$ \\
\hline \multirow{4}{*}{$\begin{array}{l}1 \mathrm{~A}_{\mathrm{g}}-\mathrm{like} \rightarrow \\
2 \mathrm{~A}_{\mathrm{g}} \text {-like }\end{array}$} & I & 1.644 & 1.526 & 0.611 & 0.0004 & 0.099 & 0.021 & $4.1115(+8)$ & $2.4(-9)$ \\
\hline & II & 1.830 & 1.698 & 0.681 & -0.004 & 0.118 & 0.039 & $4.5144(+8)$ & $2.2(-9)$ \\
\hline & III & 1.924 & -1.712 & -0.840 & 0.254 & 0.126 & 0.037 & $4.4393(+8)$ & $2.3(-9)$ \\
\hline & IV & 1.711 & 1.571 & 0.624 & -0.260 & 0.105 & 0.033 & $4.1080(+8)$ & $2.4(-9)$ \\
\hline
\end{tabular}

The values in parentheses are the powers to the base 10 . 
Table 4. Vertical excitation energies from the ground state to the first excited state at different geometries.

\begin{tabular}{lccc}
\hline & & \multicolumn{2}{c}{ Vertical excitation energy $(\mathrm{eV})$} \\
\cline { 3 - 4 } Transition & Compound & $\left(180^{\circ}, 180^{\circ}\right)$ & $\left(90^{\circ}, 180^{\circ}\right)$ \\
\hline $\mathrm{S}_{0} \rightarrow \mathrm{S}_{1}$ & I & 3.30 & 0.32 \\
& & $2.09^{\mathrm{a}}, 4.99^{\mathrm{a}}$ & $0.82^{\mathrm{b}}, 0.74^{\mathrm{b}}, 0.25^{\mathrm{b}}$ \\
& & $2.91^{\mathrm{b}}, 4.72^{\mathrm{b}}$ & \\
& II & $4.04^{\mathrm{c}}$ & - \\
& III & 3.34 & 0.97 \\
& IV & 3.64 & - \\
\hline
\end{tabular}

${ }^{\mathrm{a}}[25],{ }^{\mathrm{b}}[29],{ }^{\mathrm{c}}[30]$

roughly $375 \mathrm{~nm}$ for compound $\mathbf{I}$. Theoretically reported values $^{25}$ lie between $\sim 250 \mathrm{~nm}$ at MRCI/6-31G* level and $591 \mathrm{~nm}$ at AM1/CISD level of theory. In addition, MNDO/CI level predicts a value of $450 \mathrm{~nm}$. Our present value at PM3/CI level lies in between the two extreme values. Excitation energy of this molecule at $\left(90^{\circ}, 180^{\circ}\right)$ geometry is found to be $\sim 0.32 \mathrm{eV}$. Though this value is underestimated in comparison to the MRSDCI $(0.82 \mathrm{eV})^{25}$ and SDCI $(0.74 \mathrm{eV})^{29}$ predicted values, it should be remembered that these two above mentioned methods include huge amount of sizeconsistency errors $(\sim 0.35 \mathrm{eV})$. The vertical excitation energy (VEE) value at the $\left(180^{\circ}, 180^{\circ}\right)$ geometry increases with introduction of electron-donating groups on nitrogen (table 4). This value increases by almost $0.3 \mathrm{eV}$ from I to III; however the methyl-substituted species shows a slight increase $(\sim 0.04 \mathrm{eV})$ in the VEE value from the unsubstituted ion (I). This is according to our expectation as groups with $+\mathbf{I}$ effect stabilizes the ground state and destabilizes the excited state. It is already mentioned that this is also responsible for the shifting of the TICT CI position, away from the $90^{\circ}$ value of $\Gamma_{3}$.

\section{Conclusion}

Semiempirical and ab initio studies have explored the variations in the spectroscopic properties due to the introduction of electron-donating and electronwithdrawing groups by substituting a proton on the iminium nitrogen of the 2, 4-pentadieniminium cation. Presence of groups with strong $+\mathrm{I}$ effect stabilizes the ground state and increases the energy of the first excited state. This is responsible for increasing the VEE value and shifting of their TICT conical intersection position, which is comparable to the introduction of a counter ion on the $\mathrm{N}$-head position, already reported by other groups. In consequence, the ultrafast cis-trans isomerization of the corresponding retinal Schiff base analogues is likely to be affected. Several important spectroscopic parameters related to the low-lying transitions, such as transition moment, oscillator strength are reported for the first time for the four compounds studied. Einstein's coefficient values have predicted an increase of $10^{-3} \mathrm{~s}$ in the radiative lifetime value from the first to the second excited singlet state. Energy gap between these two states is found to have least value in the isopropyl compound. Thus, it can be concluded that the photochemical properties of these model compounds can be tuned by proper choice of substituents on the nitrogen, and consequently, the enhanced or reduced photoisomerization processes may lead to some significant changes in the bio-optical activities of the corresponding retinal PSB analogues.

\section{References}

1. Stoeckenius W and Rowen R 1962 J. Cell Biol. 3465

2. Wald G 1968 Science $\mathbf{1 6 2} 230$

3. Fodor S P A, Ames J B, Gebhard R, van den Berg E M M, Stoeckenius W, Lugtenburg J and Mathies R A 1988 Biochemistry 277097

4. Schulten K and Tavan P 1978 Nature 27285

5. Schulten K 1978 Energetics and structure of halophilic microorganism (eds) S R Caplan and M Ginzburg (Amsterdam: Elsevier) pp 331

6. Gerwert K and Siebert F 1986 J.EMBO 4805

7. Sekharan S, Weingart O and Buss V 2006 Biophys. J.: Biophys. Lett. L07

8. Send R and Sundholm D 2008 J. Mol. Model 14717

9. Gascón J A, Sproviero E M and Batista V S 2005 J. Chem. Theory Comput. 1674

10. Gascón J A, Sproviero E M and Batista V S 2006 Acc. Chem. Res. 39184

11. Smith S O, Myers A B, Pardoen J A, Winkel C, Mulder P P J, Lugtenburg J and Mathies R A 1984 Proc. Natl. Acad. Sci. USA $\mathbf{8 1} 2055$ 
12. Smith S O, Myers A B, Mathies R A, Pardoen J A, Winkel C, Van Den Berg E M M and Lugtenburg J 1985 Biophys. J. 47653

13. Hudson B S and Birge R R 1999 J. Phys. Chem. A 103 2274

14. Buda F, Giannozzi P and Mauri F 2000 J. Phys. Chem. B 1049048

15. Elia G R, Childs R F, Britten J F, Yang D S C and Santarsiero B D 1996 Can. J. Chem. 74591401

16. Mathies R A, Cruz C H B, Pollard W T and Shank C V 1988 Science 240777

17. Dobler J, Zinth W, Kaiser W and Oesterhelt D 1988 Chem. Phys. Lett. 144215.

18. Zinth W and Oesterhelt D 1991 Photobiology (ed) E Ricklis (New York: Plenum Press) pp. 531

19. Arlt T, Schmidt S, Zinth W, Haupts U and Oesterhelt D 1995 Chem.Phys. Lett. 241559

20. Xu D, Martin C and Schulten K 1996 Biophys. J. 70453

21. Norton J E and Houk K N 2006 Mol. Phys. 104 993

22. Garavelli M 2006 Theor. Chem. Acc. 11687

23. Cembran A, Bernardi F, Olivucci M and Garavelli M 2005 Proc. Natl. Acad. Sci. 1026255

24. Ruiz D S, Cembran A, Garavelli M, Olivucci M and Fu W 2002 Photochem. Photobiol. 76622

25. Dobado J A and Nonella M 1996 J. Phys. Chem. 100 18282
26. Conti I, Bernardi F, Orlandi G and Garavelli M 2006 Mol. Phys. 104915

27. Conti I and Garavelli M 2007 J. Photochem. Photobiol. A: Chemistry 190258

28. Ertl P 1990 Collect. Czech. Chem. Commun. 552874

29. Martin C H and Birge R R 1998 J. Phys. Chem. A 102 852

30. Tavernelli I, Röhrig U F and Rothlisberger U 2005 Mol. Phys. 103963

31. Stewart J J P 1989 J. Comput. Chem. 10209

32. Stewart J J P 1989 J. Comput. Chem. 10221

33. Schmidt $M \mathrm{~W}$, Baldridge $\mathrm{K} \mathrm{K}$, Boatz J A, Elbert S T, Gordon M S, Jensen J J, Koseki S, Matsunaga N, Nguyen K A, Su S, Windus T L, Dupuis M and Montgomery J A 1993 J. Comput. Chem. 141347

34. Brooks B and Schaefer H F 1979 J. Chem. Phys. 705092

35. Brooks B, Laidig W, Saxe P, Handy N and Schaefer H F 1980 Phys. Scripta 21312

36. Chattopadhyay A 2011 J. Phys. B: At. Mol. Opt. Phys. 44165101

37. Chattopadhyay A 2010 J. Chem. Sci. 122259

38. Davidson E R 1975 J. Comput. Phys. 1787

39. Elbert S T 1987 Theoret. Chim. Acta 71169

40. Weinhold F 1970 J. Chem. Phys. 541874

41. Bauschlicher C W and Langhoff S R 1991 Theoret. Chim. Acta 7993

42. Koseki S and Gordon M S 1987 J. Mol. Spectrosc. 123392 